\title{
PERIODIC SOLUTIONS OF SINGULAR DIFFERENTIAL EQUATIONS WITH SIGN-CHANGING POTENTIAL
}

\author{
JIFENG CHU ${ }^{凶}$ and ZIHENG ZHANG
}

(Received 3 May 2009)

\begin{abstract}
In this paper we study the existence of positive periodic solutions to second-order singular differential equations with the sign-changing potential. Both the repulsive case and the attractive case are studied. The proof is based on Schauder's fixed point theorem. Recent results in the literature are generalized and significantly improved.
\end{abstract}

2000 Mathematics subject classification: primary 34C25; secondary 34D20.

Keywords and phrases: periodic solution, singular differential equation, Schauder's fixed point theorem, sign-changing potential.

\section{Introduction}

In this paper we study the existence of positive $T$-periodic solutions of the secondorder differential equation

$$
x^{\prime \prime}+a(t) x=f(t, x)+e(t),
$$

where $a(t), e(t)$ are continuous and $T$-periodic functions. The potential $f(t, x)$ is continuous in $(t, x), T$-periodic in $t$ and may be singular at $x=0$.

From the physical explanation, we say that (1.1) has a repulsive singularity at $x=0$ if

$$
\lim _{x \rightarrow 0^{+}} f(t, x)=+\infty, \quad \text { uniformly in } t,
$$

whereas (1.1) has an attractive singularity at $x=0$ if

$$
\lim _{x \rightarrow 0^{+}} f(t, x)=-\infty, \quad \text { uniformly in } t .
$$

Jifeng Chu is supported by the National Natural Science Foundation of China (Grant No. 10801044), Jiangsu Natural Science Foundation (Grant No. BK2008356), the Program for New Century Excellent Talents in University (Grant No. NCET-10-0325) and the Fundamental Research Funds for the Central Universities.

(C) 2010 Australian Mathematical Publishing Association Inc. 0004-9727/2010 \$16.00 
Beginning with the paper of Lazer and Solimini [20], the semilinear singular differential equation

$$
x^{\prime \prime}+a(t) x=\frac{b(t)}{x^{\lambda}}+e(t),
$$

with $a, b, e \in \mathbb{C}[0, T]$ and $\lambda>0$, has attracted the attention of many researchers during the last two decades $[4,5,8,19,21,22,24,25,27,29]$. Some strong force conditions introduced by Gordon [15] are standard in related earlier work [11, 12, 16, 27-29]. This condition corresponds to the case where $\lambda \geq 1$ in Equation (1.2). With a strong singularity, the energy near $x=0$ becomes infinite, and this fact is very useful for obtaining a priori bounds of periodic solutions. Compared with the case of a strong singularity, the study of the existence of periodic solutions in the presence of a weak singularity is more recent, but has also attracted many researchers $[4,7,9,10,13,14,23,24]$. Some classical tools have been used to study singular differential equations in the literature. These classical tools include the method of upper and lower solutions [1, 17], degree theory [27-29], some fixed point theorems in cones for completely continuous operators [14, 22, 23], Schauder's fixed point theorem $[8,13,24]$ and a nonlinear Leray-Schauder alternative principle $[6,9,18,19]$.

In this paper we establish the existence of positive $T$-periodic solutions to Equation (1.1) through a basic application of Schauder's fixed point theorem. Our new results generalize in several respects some results contained in [8, 9, 19, 22, 24, 25]. Our main motivation is to obtain new existence results for positive $T$-periodic solutions of the equations

$$
x^{\prime \prime}+a(t) x=b(t) \ln x+e(t)
$$

and

$$
x^{\prime \prime}+a(t) x=\frac{b(t)}{x^{\alpha}}+\mu c(t) x^{\beta}+e(t),
$$

where $a, b, c, e \in \mathbb{C}[0, T], \alpha, \beta>0$ and $\mu>0$ is a parameter. Note that, when $b \succ 0$ $(b \prec 0)$, (1.3) presents an attractive (repulsive) singularity at $x=0$, whereas (1.4) presents a repulsive (attractive) singularity at $x=0$. We deal with both the repulsive case and the attractive case. From these examples, one may readily see that one advantage of our method is that we can allow that the potential can change sign. Therefore we generalize and improve some results contained in [8, 9, 19, 22, 24]. We also emphasize that our new results are applicable to the case of a strong singularity as well as the case of a weak singularity.

The rest of the paper is organized as follows. In Section 2 some preliminary results are given. In Section 3 we state and prove the existence results when (1.1) has an attractive singularity. The existence results for the repulsive case are established in Section 4. Applications of the new results to (1.3) and (1.4) are also given in Sections 3 and 4.

Henceforth, let us denote by $p^{*}$ and $p_{*}$ the essential supremum and infimum of a given function $p \in L^{1}[0, T]$, if they exist. Also, we write $p \succ 0$ if $p \geq 0$ for almost 
every $t \in[0, T]$ and it is positive in a set of positive measure. The usual $L^{p}$-norm is denoted by $\|\cdot\|_{p}$. The conjugate exponent of $p$ is denoted by $q:(1 / p)+(1 / q)=1$.

\section{Preliminaries}

Consider the Hill equation,

$$
x^{\prime \prime}+a(t) x=0
$$

with periodic boundary conditions

$$
x(0)=x(T), \quad x^{\prime}(0)=x^{\prime}(T) .
$$

Throughout this paper we assume that the following hypothesis is satisfied.

(A) The Green function $G(t, s)$, associated with (2.1) and (2.2), is nonnegative for all $(t, s) \in[0, T] \times[0, T]$.

In other words, the anti-maximum principle holds for (2.1) and (2.2). See [26, 30]. When $a(t) \equiv k^{2}$, condition (A) is equivalent to $0<k^{2} \leq(\pi / T)^{2}$. In this case,

$$
G(t, s)= \begin{cases}\frac{\sin k(t-s)+\sin k(T-t+s)}{2 k(1-\cos k T)} & \text { for } 0 \leq s \leq t \leq T, \\ \frac{\sin k(s-t)+\sin k(T-s+t)}{2 k(1-\cos k T)} & \text { for } 0 \leq t \leq s \leq T .\end{cases}
$$

See $[14,22]$.

For a nonconstant function $a(t)$, there is an $L^{p}$-criterion proved in [22], which is given in the following lemma for the sake of completeness. Let $\mathbf{K}(q)$ denote the best Sobolev constant in the inequality

$$
C\|u\|_{q}^{2} \leq\left\|u^{\prime}\right\|_{2}^{2} \quad \text { for all } u \in H_{0}^{1}(0, T) .
$$

The explicit formula for $\mathbf{K}(q)$ is

$$
\mathbf{K}(q)= \begin{cases}\frac{2 \pi}{q T^{1+2 / q}}\left(\frac{2}{2+q}\right)^{1-2 / q}\left(\frac{\Gamma\left(\frac{1}{q}\right)}{\Gamma\left(\frac{1}{2}+\frac{1}{q}\right)}\right)^{2} & \text { if } 1 \leq q<\infty, \\ \frac{4}{T} & \text { if } q=\infty,\end{cases}
$$

where $\Gamma$ is the gamma function.

LEMMA 2.1. Assume that $a(t) \succ 0$ and $a \in L^{p}[0, T]$ for some $1 \leq p \leq \infty$. If

$$
\|a\|_{p} \leq \mathbf{K}(2 q)
$$

then the standing hypothesis (A) holds. 
REMARK 2.2. In $[4,5,14,19,22,23]$, the existence results are based on the positivity of $G(t, s)$, which plays a very important role in employing some fixed point theorems in cones for completely continuous operators and a nonlinear alternative principle of Leray and Schauder. Therefore, the results in the above mentioned papers cannot cover the critical case. Our assumption (A) only needs $G(t, s)$ to be nonnegative, and therefore our results can cover the critical case.

\section{Attractive case}

In this section, we establish the existence of positive periodic solutions of (1.1) in the attractive case. From now on, we use $\mathbb{C}_{T}$ to denote the set of all continuous $T$-periodic functions.

THEOREM 3.1. Assume that a(t) satisfies (A). Furthermore, suppose that there exist a function $b \succ 0$ and positive constants $R>r>0$ such that:

$\left(\mathrm{H}_{1}\right)$ there exists a continuous nondecreasing function $g: \mathbb{R}^{+} \rightarrow \mathbb{R}^{+}$such that

$$
\begin{gathered}
f(t, x)<0 \quad \text { for each } 0<x<r \text { and } t \in[0, T], \\
f(t, r)=0 \quad \text { uniformly in } t \in[0, T], \\
0 \leq f(t, x) \leq b(t) g(x) \text { for each } x>r \text { and } t \in[0, T]
\end{gathered}
$$

$\left(\mathrm{H}_{2}\right) \beta^{*} g(R)+\gamma^{*} \leq R$ with

$$
\gamma(t)=\int_{0}^{T} G(t, s) e(s) d s, \quad \beta(t)=\int_{0}^{T} G(t, s) b(s) d s,
$$

where $\beta^{*}$ and $\gamma^{*}$ denote the essential supremum of $\beta$ and $\gamma$, and $\gamma_{*}$ denotes the essential infimum of $\gamma$. If $\gamma_{*} \geq r$, then (1.1) has at least one positive T-periodic solution.

PROOF. A $T$-periodic solution of Equation (1.1) is just a fixed point of the completely continuous map $\mathcal{F}: \mathbb{C}_{T} \rightarrow \mathbb{C}_{T}$ defined by

$$
\begin{aligned}
(\mathcal{F} x)(t) & =\int_{0}^{T} G(t, s)[f(s, x(s))+e(s)] d s \\
& =\int_{0}^{T} G(t, s) f(s, x(s)) d s+\gamma(t) .
\end{aligned}
$$

Let $R$ be the positive constant satisfying $\left(\mathrm{H}_{2}\right)$. Now we define the set

$$
\mathcal{A}=\left\{x \in \mathbb{C}_{T}: \gamma_{*} \leq x(t) \leq R \text { for all } t \in[0, T]\right\}
$$

Obviously, $\mathcal{A}$ is a closed convex set. Next we prove that $\mathcal{F}(\mathcal{A}) \subset \mathcal{A}$.

In fact, for each $x \in \mathcal{A}$ and for all $t \in[0, T]$, using the fact that $G(t, s) \geq 0$ for all $(t, s) \in[0, T] \times[0, T]$, together with condition $\left(\mathrm{H}_{1}\right)$,

$$
(\mathcal{F} x)(t) \geq \gamma(t) \geq \gamma_{*} .
$$


On the other hand, by conditions $\left(\mathrm{H}_{1}\right)$ and $\left(\mathrm{H}_{2}\right)$,

$$
\begin{aligned}
(\mathcal{F} x)(t) & \leq \int_{0}^{T} G(t, s) b(s) g(x(s)) d s+\gamma(t) \\
& \leq \beta^{*} g(R)+\gamma^{*} \leq R .
\end{aligned}
$$

In conclusion, $\mathcal{F}(\mathcal{A}) \subset \mathcal{A}$. Moreover, it is easy to check that $\mathcal{F}: \mathcal{A} \rightarrow \mathcal{A}$ is completely continuous. By a direct application of Schauder's fixed point theorem, (1.1) has at least one positive $T$-periodic solution.

EXAMPLE 3.2. Assume that $a(t)$ satisfies (A). Let the nonlinearity in (1.1) be

$$
f(t, x)=-\frac{1}{x^{\alpha}}+\mu x^{\beta},
$$

where $\alpha>0, \beta \geq 0$ and $\mu \geq 0$ is a parameter. For each $e(t)$ with

$$
\gamma_{*} \geq \hat{\mu}=\mu^{-(1 /(\alpha+\beta))}
$$

we have the following results.

(i) If $\beta<1$, then (1.1) has at least one positive $T$-periodic solution for each $\mu \geq 0$.

(ii) If $\beta \geq 1$, then (1.1) has at least one positive $T$-periodic solution for each $0 \leq$ $\mu<\mu_{1}$, where $\mu_{1}$ is some positive constant.

PROOF. We apply Theorem 3.1. To this end, we take

$$
r=\hat{\mu}, \quad g(x)=\mu x^{\beta}, \quad b(t)=1 .
$$

Then $\left(\mathrm{H}_{1}\right)$ is satisfied. Let $\omega(t)=\int_{0}^{T} G(t, s) d s$. Now condition $\left(\mathrm{H}_{2}\right)$ becomes

$$
\mu<\frac{R-\gamma^{*}}{\omega_{*} R^{\beta}}
$$

for some $R>0$ with $R>\hat{\mu}$. So (1.1) has at least one positive $T$-periodic solution for

$$
0<\mu<\mu_{1}=\sup _{R>\hat{\mu}} \frac{R-\gamma^{*}}{\omega_{*} R^{\beta}} .
$$

Note that $\mu_{1}=\infty$ if $\beta<1$ and $\mu_{*}<\infty$ if $\beta \geq 1$. We have the desired results.

REMARK 3.3. It is easy to find results analogous to Example 3.2 for the general Equation (1.4) with $b, c \succ 0$, but the notation becomes cumbersome. Here we consider only (3.3) for simplicity.

EXAmple 3.4. Assume that $a(t)$ satisfies (A) and $b(t)$ is a positive $T$-periodic continuous function. Then for each $e(t)$ with $\gamma_{*} \geq 1$, Equation (1.3) has at least one positive $T$-periodic solution. 
PROOF. We apply Theorem 3.1. To this end, we take $r=1, g(x)=\ln x$. Then $\left(\mathrm{H}_{1}\right)$ is satisfied and condition $\left(\mathrm{H}_{2}\right)$ becomes

$$
\beta^{*} \ln R+\gamma^{*} \leq R
$$

for some $R>0$ with $R>1$. Note that $\beta^{*}>0$ and

$$
\lim _{R \rightarrow+\infty} \frac{\ln R}{R}=0
$$

and it is always possible to find such $R \gg 1$ that (3.4) is satisfied. Now we have the result using Theorem 3.1.

\section{Repulsive case}

In this section, we establish the existence of positive periodic solutions of (1.1) in the repulsive case.

THEOREM 4.1. Assume that a(t) satisfies (A). Furthermore, suppose that there exist a function $b \succ 0$ and a constant $R>0$ such that:

$\left(\mathrm{H}_{3}\right)$ there exists a continuous nonincreasing function $g: \mathbb{R}^{+} \rightarrow \mathbb{R}^{+}$such that

$$
0<f(t, x) \leq b(t) g(x) \quad \text { for all } 0<x<R \text { and } t \in[0, T],
$$

and

$$
f(t, x) \leq 0 \quad \text { for } x \geq R \text { and } t \in[0, T]
$$

$\left(\mathrm{H}_{4}\right) R \geq g\left(\gamma_{*}\right) \beta^{*}+\gamma^{*}$.

If $\gamma_{*}>0$, then (1.1) has at least one positive T-periodic solution.

PROOF. We follow the same strategy and notation as in the proof of Theorem 3.1. Let $R$ be the positive constant satisfying $\left(\mathrm{H}_{4}\right)$. Then $R>\gamma_{*}>0$ since $R>\gamma^{*}$. Next we prove $\mathcal{F}(\mathcal{A}) \subset \mathcal{A}$.

For each $x \in \mathcal{A}$ and for all $t \in[0, T]$, by the nonnegative sign of $G(t, s)$ and $f(t, x)$,

$$
(\mathcal{F} x)(t)=\int_{0}^{T} G(t, s) f(s, x(s)) d s+\gamma(t) \geq \gamma_{*}>0 .
$$

On the other hand, using $\left(\mathrm{H}_{3}\right)$ and $\left(\mathrm{H}_{4}\right)$,

$$
\begin{aligned}
(\mathcal{F} x)(t) & =\int_{0}^{T} G(t, s) b(s) g(x(s)) d s+\gamma(t) \\
& \leq g\left(\gamma_{*}\right) \beta^{*}+\gamma^{*} \leq R .
\end{aligned}
$$

In conclusion, $\mathcal{F}(\mathcal{A}) \subset \mathcal{A}$ and the proof is finished by Schauder's fixed point theorem. 
EXAMPLE 4.2. Assume that $a(t)$ satisfies (A). Let the nonlinearity in (1.1) be

$$
f(t, x)=\frac{1}{x^{\alpha}}-\mu x^{\beta},
$$

where $\alpha>0, \beta \geq 0$ and $\mu \geq 0$ is a parameter. Then there exists a positive constant $\mu_{2}$ such that (1.1) has at least one positive $T$-periodic solution for each $0 \leq \mu<\mu_{2}$.

PROOF. We apply Theorem 4.1. To this end, we take

$$
R=\hat{\mu}=\mu^{-(1 /(\alpha+\beta))}, \quad g(x)=\frac{1}{x^{\alpha}}, \quad b(t)=1 .
$$

Then $\left(\mathrm{H}_{3}\right)$ is satisfied and condition $\left(\mathrm{H}_{4}\right)$ becomes

$$
R \geq \gamma_{*}^{-\alpha} \beta^{*}+\gamma^{*}
$$

for some $R>0$ with $R=\hat{\mu}$. It is always possible to find $\mu_{2} \ll 1$ such that (4.2) is satisfied for each $0 \leq \mu<\mu_{2}$.

EXAmple 4.3. Assume that $a(t)$ satisfies (A) and $b \succ 0$ is a $T$-periodic continuous function. Then

$$
x^{\prime \prime}+a(t) x=-b(t) \ln x+e(t)
$$

has at least one positive $T$-periodic solution if

$$
\gamma_{*}>0, \quad \gamma^{*} \leq 1+\beta^{*} \ln \gamma_{*} .
$$

PROOF. We take $g(x)=-\ln x$ and $R=1$. Then $\left(\mathrm{H}_{3}\right)$ is satisfied and condition $\left(\mathrm{H}_{4}\right)$ just reads as (4.4).

REMARK 4.4. We emphasize that our new results in this paper are applicable to the case of a strong singularity as well as the case of a weak singularity. Moreover, $e$ does not need to be positive.

REMARK 4.5. By the recent results proved in [2], we can improve all results in Sections 3 and 4, by allowing $a(t)$ to change sign. In particular, we impose an integral condition $\int_{0}^{T} a(t) d t>0$ instead of $a \succ 0$. In this case, the corresponding criterion in Lemma 2.1 becomes $\left\|a_{+}\right\|_{p} \leq \mathbf{K}(2 q)$.

REMARK 4.6. All results in this paper are also applicable to the more general differential equation

$$
\left(b(t) x^{\prime}\right)^{\prime}+a(t) x=f(t, x)+e(t),
$$

assuming that the Green function $G(t, s)$, associated with the corresponding linear equation, is nonnegative for all $(t, s) \in[0, T] \times[0, T]$, because the problem of finding a $T$-periodic solution of system (4.5) is expressed as a fixed point problem for the same operator defined in (3.1). When $b(t)>0$, a criterion similar to Lemma 2.1 has recently been established in [3]. This criterion states that

$$
\left\|a b^{1 / q}\right\|_{p} \leq \mathbf{K}(2 q),(1<p \leq+\infty) \quad \text { or }\|a\|_{1} \cdot\|1 / b\|_{1} \leq 4 .
$$




\section{References}

[1] D. Bonheure and C. De Coster, 'Forced singular oscillators and the method of lower and upper solutions', Topol. Methods Nonlinear Anal. 22 (2003), 297-317.

[2] A. Cabada and J. A. Cid, 'On the sign of the Green's function associated to Hill's equation with an indefinite potential', Appl. Math. Comput. 205 (2008), 303-308.

[3] J. Chu and Y. H. Lee, 'The sign of Green's functions and a uniqueness result for a weak singular equation', Preprint.

[4] J. Chu and M. Li, 'Positive periodic solutions of Hill's equations with singular nonlinear perturbations', Nonlinear Anal. 69 (2008), 276-286.

[5] J. Chu, X. Lin, D. Jiang, D. O'Regan and P. R. Agarwal, 'Multiplicity of positive solutions to second order differential equations', Bull. Aust. Math. Soc. 73 (2006), 175-182.

[6] J. Chu and J. J. Nieto, 'Impulsive periodic solutions of first-order singular differential equations', Bull. London Math. Soc. 40 (2008), 143-150.

[7] J. Chu and J. J. Nieto, 'Recent existence results for second-order singular periodic differential equations', Bound. Value Probl. 540863 (2009), 1-20.

[8] J. Chu and P. J. Torres, 'Applications of Schauder's fixed point theorem to singular differential equations', Bull. London Math. Soc. 39 (2007), 653-660.

[9] J. Chu, P. J. Torres and M. Zhang, 'Periodic solutions of second order non-autonomous singular dynamical systems', J. Differential Equations 239 (2007), 196-212.

[10] J. Chu and M. Zhang, 'Rotation numbers and Lyapunov stability of elliptic periodic solutions', Discrete Contin. Dyn. Syst. 21 (2008), 1071-1094.

[11] M. del Pino and R. Manásevich, 'Infinitely many $T$-periodic solutions for a problem arising in nonlinear elasticity', J. Differential Equations 103 (1993), 260-277.

[12] A. Fonda, R. Manásevich and F. Zanolin, 'Subharmonic solutions for some second order differential equations with singularities', SIAM J. Math. Anal. 24 (1993), 1294-1311.

[13] D. Franco and P. J. Torres, 'Periodic solutions of singular systems without the strong force condition', Proc. Amer. Math. Soc. 136 (2008), 1229-1236.

[14] D. Franco and J. R. L. Webb, 'Collisionless orbits of singular and nonsingular dynamical systems', Discrete Contin. Dyn. Syst. 15 (2006), 747-757.

[15] W. B. Gordon, 'Conservative dynamical systems involving strong forces', Trans. Amer. Math. Soc. 204 (1975), 113-135.

[16] P. Habets and L. Sanchez, 'Periodic solution of some Liénard equations with singularities', Proc. Amer. Math. Soc. 109 (1990), 1135-1144.

[17] P. Habets and F. Zanolin, 'Upper and lower solutions for a generalized Emden-Fowler equation', J. Math. Anal. Appl. 181 (1994), 684-700.

[18] D. Jiang, J. Chu, D. O'Regan and R. P. Agarwal, 'Multiple positive solutions to superlinear periodic boundary value problems with repulsive singular forces', J. Math. Anal. Appl. 286 (2003), 563-576.

[19] D. Jiang, J. Chu and M. Zhang, 'Multiplicity of positive periodic solutions to superlinear repulsive singular equations', J. Differential Equations 211 (2005), 282-302.

[20] A. C. Lazer and S. Solimini, 'On periodic solutions of nonlinear differential equations with singularities', Proc. Amer. Math. Soc. 99 (1987), 109-114.

[21] I. Rachunková, M. Tvrdý and I. Vrkoč, 'Existence of nonnegative and nonpositive solutions for second order periodic boundary value problems', J. Differential Equations 176 (2001), 445-469.

[22] P. J. Torres, 'Existence of one-signed periodic solutions of some second-order differential equations via a Krasnoselskii fixed point theorem', J. Differential Equations 190 (2003), 643-662.

[23] P. J. Torres, 'Non-collision periodic solutions of forced dynamical systems with weak singularities', Discrete Contin. Dyn. Syst. 11 (2004), 693-698.

[24] P. J. Torres, 'Weak singularities may help periodic solutions to exist', J. Differential Equations 232 (2007), 277-284.

[25] P. J. Torres, 'Existence and stability of periodic solutions for second order semilinear differential equations with a singular nonlinearity', Proc. Roy. Soc. Edinburgh Sect. A 137 (2007), 195-201. 
[26] P. J. Torres and M. Zhang, 'A monotone iterative scheme for a nonlinear second order equation based on a generalized anti-maximum principle', Math. Nachr. 251 (2003), 101-107.

[27] P. Yan and M. Zhang, 'Higher order nonresonance for differential equations with singularities', Math. Methods Appl. Sci. 26 (2003), 1067-1074.

[28] M. Zhang, 'A relationship between the periodic and the Dirichlet BVPs of singular differential equations', Proc. Roy. Soc. Edinburgh Sect. A 128 (1998), 1099-1114.

[29] M. Zhang, 'Periodic solutions of equations of Emarkov-Pinney type', Adv. Nonlinear Stud. 6 (2006), 57-67.

[30] M. Zhang and W. Li, 'A Lyapunov-type stability criterion using $L^{\alpha}$ norms', Proc. Amer. Math. Soc. 130 (2002), 3325-3333.

JIFENG CHU, Department of Mathematics, College of Science, Hohai University, Nanjing 210098, PR China

e-mail: chujf05@mails.tsinghua.edu.cn

ZIHENG ZHANG, School of Mathematical Sciences, Beijing Normal University, Beijing 100875, PR China

e-mail: zhzh@mail.bnu.edu.cn 